\title{
La discrimination systémique dans le système éducatif français
}

Une étude de cas sur l'impact des conditions sociales de certification dans l'orientation des enfants des minorités ethniques d'un « collège de banlieue »

\section{Philippe Perrot}

\section{(2) OpenEdition}

\section{Journals}

Édition électronique

URL : http://journals.openedition.org/urmis/259

ISSN : $1773-021 \mathrm{X}$

Éditeur

Urmis

Édition imprimée

Date de publication : 1 décembre 2006

ISSN : 1287-471X

Référence électronique

Philippe Perrot, "La discrimination systémique dans le système éducatif français », Cahiers de l'Urmis [En ligne], 10-11 | décembre 2006, mis en ligne le 15 décembre 2006, consulté le 07 septembre 2020 URL : http://journals.openedition.org/urmis/259

Ce document a été généré automatiquement le 7 septembre 2020

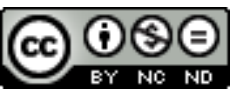

Les contenus des Cahiers de l'Urmis sont disponibles selon les termes de la Licence Creative Commons Attribution - Pas d'Utilisation Commerciale - Pas de Modification 4.0 International. 


\title{
La discrimination systémique dans le système éducatif français
}

\author{
Une étude de cas sur l'impact des conditions sociales de certification \\ dans l'orientation des enfants des minorités ethniques d'un « collège de \\ banlieue »
}

Philippe Perrot

\section{Introduction}

1 Comme le remarque Patrick Simon (2005), la question des discriminations a subi une transformation assez sensible en France ces dernières années. L'accumulation d'enquêtes convergentes a en effet quelque peu dissipé une perspective qu'on peu qualifier, avec le recul, de "grossièrement morale ». L'approche du racisme s'en est trouvée, elle même, renouvelée: désindexée de la lutte idéologique contre les groupements racistes, elle peut se recentrer sur « cette somme de petites décisions, de comportements ou d'appréciations qui, enchaînées et répétées de façon routinières quasiment invisibles, composent un système dense d'actes discriminatoires et empêchent l'accès plein et entier à la jouissance des droits d'individus définis par leurs origines ethniques et raciales. » (p. 1).

2 Comme l'avance plus loin l'auteur (p. 2), cette définition implique de faire pivoter l'essentiel du regard des principes aux conséquences: il ne s'agit plus de partir du discours pour aboutir à ses effets, mais de remonter des effets à des causes dont on ne sait pas a priori quelles formes elles empruntent.

3 Vu sous cet angle, le déplacement semble important. «Discrimination » ne semble ainsi plus pointer une classe relativement restreinte de phénomènes dont la familiarité semblait acquise antérieurement au travail d'enquête.

4 Pourtant, il n'est pas du tout sûr que le concept soit expurgé autant que possible de ce que Passeron (1982) appelait «sa capacité de désignation directe ». A lire dans le texte cité, la définition de la discrimination indirecte de l'Union européenne servant de base à l'argumentation, on cherche « [...] une disposition, un critère, une pratique [...]». Les causes sont ici singulières et mutuellement exclusives. Il semble au premier abord qu'on ne puisse pas trouver des dispositions, des critères, des pratiques ou, pire, un 
enchevêtrement pluriel de ces trois éléments. Ainsi, la «simplicité » présupposée des processus en cause nous semble être un bonne indication de la persistance des attentes que les discriminations doivent prendre une forme reconnaissable, en deçà du travail de description et de mesure. On ne s'attend plus à trouver directement un idiome raciste. Mais on s'attend toujours à une certaine dose de familiarité : à ce que la réalité, des discours ou des actes, se résolvent en une interconnexion de moments ayant chacun leur portée propre.

5 Ce faisant, on réintroduit la figure (certes clarifiée) d'une intention sous-jacente. Sans doute, la nature discriminatoire des actes n'apparaît plus au premier abord. Ils sont « apparemment neutres» (p. 1), «quasiment invisibles» (ibid.) et demandent à être "révélés» (p. 8). Sans doute également, cette "révélation» n'est pas facile. L'impression néanmoins demeure qu'il s'agit toujours de faire "tomber le masque ", même si celui-ci est plus épais et que sa chute nécessite plus de moyens, notamment statistiques, que dans sa version " grossièrement morale ».

6 Congédiée à un niveau programmatique, l'intentionnalité hante ainsi le propos pour réapparaître sous une forme qui ressemble à quelque chose comme une sécularisation. Les habitudes prises découlent ainsi de toutes les façons des « formations historiques des sociétés postcoloniales et post-migratoires. » (p. 8, souligné par nous).

7 Se donne-t-on ainsi des chances d'analyser des discriminations vraiment systémiques? Car la différence entre la discrimination directe et indirecte est moins alors une différence de nature qu'une différence de degré dans la complexité organisationnelle. Il s'agirait alors de réarmer le regard pour retrouver le bon réagencement des parties plutôt que de changer profondément de perspective.

8 Le texte qui suit sur le processus d'orientation des élèves de troisième d'un collège périphérique tente de donner un aperçu de ce que pourrait être ce changement de perspective : trouver le système dans la discrimination plutôt qu'un système (même "dense ») d'actes discriminatoires. Ainsi nous espérons mettre en évidence que cette discrimination, si elle peut être établie, n'offre néanmoins ni moments intrinsèquement signifiants ( $\mathrm{du}$ moins dans la partie ethnique), ni intentionnalité maîtresse, qu'elle soit sécularisée ou pas.

9 Mais avant cela, il nous faut revenir sur les raisons qui nous ont fait nous intéresser à la discrimination au sein du système éducatif français.

2. Le problème

10 C'est un euphémisme de dire aujourd'hui que l'existence de discriminations au sein du système éducatif français ne fait pas débat dans le champ sociologique.

2.1. Le constat d'un non-débat

11 En fait, comme souvent dans les questions migratoires en France, le constat à l'origine de ce non-débat n'a été établi qu'incidemment.

12 On le doit pour beaucoup à l'enquête menée par Vallet et Caille (1996), «Les élèves étrangers ou issus de l'immigration dans l'école et le collège français; une étude d'ensemble ». Trouver d'éventuelles discriminations n'était pas le but affiché par ces auteurs. Leur perspective était essentiellement descriptive et entrait explicitement dans la thématique de l'intégration. Dans ce cadre, la question «les immigrés s'intègrent-il ? » revenait à la question « leurs descendants ont-ils une réussite scolaire comparable aux enfants autochtones?» 
13 La conclusion obtenue à l'aide d'analyses de régressions logistiques était franchement opposée à la vision «bien trompeuse» du sens commun. Les jeunes issus de l'immigration avaient ainsi une "réussite scolaire ", c'est-à-dire un taux d'accès en seconde générale et technologique à l'issue de la troisième, supérieur aux enfants français ou d'origine française.

14 Il n'est pas besoin de trop forcer son imagination pour voir l'impact implicite d'un tel constat. S'il est admis tel quel, la recherche sociologique peut difficilement parler de discriminations. Sans conséquences, les études nombreuses et détaillées sur l'utilisation effective de catégories ethniques au sein du système éducatif ne débouchent que sur elles-mêmes (ce qui n'est pas si mal), tandis que la discrimination indirecte se voit privée de tout point de départ.

15 Heureusement, pourrait-on dire, il existe de très bonnes raisons pour ne pas admettre tel quel ce résultat. Dans leur critique globale des enquêtes du même type, celles de la sociologie de la "démocratisation scolaire ", Garcia et Poupeau (2003) pointent trois biais importants.

16 Passons très vite sur la critique récurrente (bien que fondée et importante) sur les limites de l'analyse multivariée. L'idée selon laquelle ce type d'analyse prétend apporter une clarification factuelle en construisant des situations pseudoexpérimentales ayant, paradoxalement, une faible probabilité réelle d'exister est suffisamment répandue pour ne pas y revenir longuement.

Le deuxième biais est aussi connu. Définir la réussite scolaire comme l'accès ou pas en seconde générale et technologique ne prend pas en compte le fait que le hiérarchisation croissante des filières est considérée comme une composante fondamentale de l'étude des inégalités face à l'école. Une seconde qui conduit à un bac $\mathrm{S}$ option mathématiques ou à un bac STT, ne devrait sans doute pas être traitée comme la même seconde.

18 Enfin, et c'est ce qui nous intéresse en premier lieu, Sandrine Garcia et Franck Poupeau pointent ce qu'ils appellent l'oubli des conditions sociales de certification. L'idée en est que, quelles que soient les normes et les règles édictées par l'institution (ce que Pierre Merle (1996) appelle « le paradigme évaluatif »), celles-ci subissent une réappropriation par les acteurs locaux en fonction du contexte de leur application.

19 En particulier, cela veut dire que le niveau de compétences scripturales-scolaires ${ }^{1}$ réellement acquises par les élèves est très dépendant de l'inscription des établissements dans leur environnement socio-économique. En outre, il est fort probable que ces arrangements pédagogiques soit probablement recouverts, tout ou en partie, par des arrangements évaluatifs. De la même manière qu'on adapte les programmes à l'idée qu'on se fait des « capacités » du public à les absorber, on adapte également la notation sous la forme de biais sociaux. Bien plus qu'un instrument neutre de classification, le jeu entre la note attribuée et celle que l'habitus évaluatif serait tout prêt à accorder est très souvent une ressource dans les dialogues que le professeur entretient avec différentes catégories de personnes: les élèves, les parents, les membres de l'administration, l'encadrement.

20 Si on prend en compte le fait, premièrement, que le collège est un type d'établissement particulièrement touché par les effets de contexte ${ }^{2}$ et, deuxièmement, que les jeunes issus de l'immigration y sont également particulièrement sensibles à cause de la ségrégation scolaire qui les touche ${ }^{3}$, on arrive à la conclusion suivante : un ensemble de 
procédures formellement neutres ethniquement au collège et saisies comme telles à l'aide d'indicateurs «standard " peut aboutir à des situations clairement contrastées au lycée. En ce sens, il est tout à fait possible que l'enquête en question, en dépit d'un raisonnement "toutes choses égales par ailleurs" particulièrement poussé ne renseigne finalement que faiblement sur le sort concret des enfants des minorités ethniques.

21 Le but de cet article est de donner un aperçu de l'impact des conditions sociales de certification dans la carrière scolaire des enfants de migrants. Il prend appui sur la description fine d'un processus d'orientation des élèves de troisième d'un «collège de banlieue ». Il se centre principalement sur les biais sociaux de notation avec l'hypothèse qu'ils résument à eux seuls une grande part des différents arrangements au sein du collège.

\subsection{Biais sociaux de notation, biais sociaux d'orientation}

L'étude des biais sociaux de notation ne semblent pas avoir beaucoup inspiré les sociologues. On va le voir, la difficulté est d'abord d'ordre méthodologique. Néanmoins, on peut rapidement dire que deux raisons semblent à l'origine de ce désintérêt. D'une part, souvent évaluateurs eux mêmes, ils ont sans doute beaucoup de mal à prendre la mesure intuitive de leur importance. Or, celle-ci est considérable. Comme le rappelle Pierre Merle (1996), une double correction de copies opérée pour le baccalauréat de 1984 sur deux académies a montré que des écarts de 10 points (sur 20) n'étaient absolument par rares. D'autre part, confrontés à l'«effet établissement», les chercheurs ont eu souvent tendance à raisonner avec des concepts de haut niveau, tel que la «mobilisation enseignante $»^{4}$, plutôt que de se concentrer sur une description fine des modalités concrètes du fonctionnement institutionnel.

Effectivement, la difficulté principale des biais sociaux de notation est que, quand on reste au niveau de l'établissement, leur mise en évidence requiert a priori une notation de référence permettant de mesurer l'écart, le "delta ", avec la notation effective. Or ces évaluations normalisées n'existaient pas au niveau du palier d'orientation de troisième. En concevoir, comme l'avaient fait Duru-Bellat et Mingat (1993), restait une option hors de notre portée. En outre nous n'avons pu accéder aux notes obtenues lors des épreuves écrites du brevet des collèges. Dès lors, mettre en évidence le jeu de la notation représentait un défi que nous n'avons pu relever qu'en mobilisant le maximum de la panoplie méthodologique offerte au sociologue. Deux pôles donc. Un pôle ethnographique avec une présence de longue haleine sur les lieux (classes, circulations, sphère administrative), de fréquentes discussions avec les personnels, une présence et un enregistrement des conseils de classe du premier et du second trimestres (nous n'avons pu, pour différentes raisons, accéder à ceux du troisième). Un pôle statistique ensuite dans lequel nous avons utilisé des outils «basiques » (tableaux de contingence), mais aussi des outils plus sophistiqués comme des analyses de régression logistiques.

Ceci établi, restituer l'ensemble de ces résultats dans un article de cet ordre est hors de question pour des raisons qu'il n'est pas difficile de comprendre. Notre restitution restera donc indicative, et nous avons choisi de nous laisser guider par les biais sociaux d'orientation. La ventilation des élèves vers les filières post-collège se fait-elle selon des principes méritocratiques? Que ce soit le cas ou pas, l'ensemble des arguments recueillis pour justifier tel ou tel cas particulier a laissé clairement apparaître des motifs qui, s'ils ne prétendent pas à l'administration complète de la preuve, en sont une 
version raisonnablement approchée. Nous commençons donc par l'orientation des élèves en fin de troisième dans un collège dit « de banlieue ».

3. Le collège avant le lycée

25 Le collège Herman Melville est un collège qui a connu une évolution très semblable à d'autres établissements dits de relégation. Placé en Zep dès la création de cette catégorie, sa population va chuter très sérieusement à partir de la fin des années 80 , passant de presque 750 élèves à un peu moins de 500 actuellement. Durant cette période, la forte évasion professorale a progressivement polarisé le corps enseignant en « vieux » et « jeunes » professeurs, ces derniers étant affectés d'un fort turn over.

3.1. Un « collège de banlieue »

Les élèves ont également beaucoup changé. Nous le savons assez précisément car nous avons pu nous reporter à une enquête menée dans cet établissement par Jean-Pierre Zirotti (1984) au milieu des années 80. Le caractère précurseur de ces travaux permet une comparabilité directe, aussi bien du point de vue des catégories socioprofessionnelles que du point de vue ethnique. En effet, tout comme dans notre cas, les élèves avaient été alors classés selon le lieu de naissance du père et pas seulement selon leur nationalité. De sorte que les trois catégories que nous avons retenues («maghrébine », «étrangère », «française ") trouvent directement leur équivalence.

On sait qu'une des hantises des études sur la scolarisation des jeunes issus de l'immigration a toujours été de neutraliser l'effet de la classe sociale d'origine. Cette opération, souvent considérée comme un pré-requis expérimental "allant-de-soi ", peut être largement questionnée. Reste que, dans le cas contraire, la population du collège nous aurait facilité grandement les choses. La césure principale qu'on rencontrait il y a une dizaine d'années entre enfants d'employés et enfants d'ouvriers ( $35 \%$ et $40 \%$ ) n'existe plus en effet aujourd'hui, tout comme ne sont plus présents les $8 \%$ de «professions intermédiaires" et les $3 \%$ de "professions intellectuelles supérieures". En fait, le concept même de PCS (profession et catégorie socioprofessionnelle) n'a pas vraiment de sens ici. La profession la plus élevée à laquelle nous avons eu affaire était celle d'une seule personne aide-soignante. Presque $60 \%$ des autres parents sont ouvriers, $30 \%$ se déclarent «sans profession » tandis que $12 \%$ se déclarent « au chômage ». Enfin, un seul individu se déclare artisan maçon.

Cette prolétarisation du public est allée de pair avec un déplacement du centre de gravité ethnique. En 1984, les enfants dont le père était né en France représentaient $85 \%$ des élèves tandis qu'ils étaient $8,5 \%$ à avoir un père né au Maghreb et $2 \%$ dans un pays d'Europe du sud. Aujourd'hui, les rapports sont presque inversés. Si on ajoute les $59 \%$ de pères maghrébins aux $17 \%$ de pères étrangers, on obtient $76 \%$ de jeunes issus de l'immigration contre $24 \%$ de « Français ».

Bref, l'ensemble des caractéristiques de Melville font de ce collège un «abcès de fixation", une figure presque caricaturale de la manière dont certaines logiques institutionnelles couplées à une dynamique périurbaine tend à homogénéiser à l'extrême et «vers le bas » les publics. Il est pour cela le candidat idéal à la mise en évidence des effets des conditions sociales de certification.

\subsection{Un modèle d'entendement professoral}

30 Pour mettre en évidence les biais d'orientation, point d'entrée au biais de notation, nous avons utilisé une analyse de régression logistique. On sait qu'un modèle logistique permet de mettre en correspondance une variable «à expliquer » de structure binaire 
(dans notre cas le passage ou pas en seconde générale et technologique) et un ensemble de variables "explicatives " qui sont en général de type catégoriel (par exemple la profession du père, de la mère, leurs pays de naissance, etc.). L'intérêt affiché de cette méthode est qu'elle permet de mettre en évidence le poids causal de chaque variable explicative indépendamment des autres variables. Par exemple, séparer dans la sousréussite scolaire "apparente" des jeunes issus de l'immigration ce qui revient en propre à l'origine ethnique de ce qui découle de l'appartenance des parents à des catégories sociales modestes.

Comme dit plus haut, ce type d'utilisation pose néanmoins de nombreux problèmes. C'est pour cela que nous avons choisi d'utiliser l'analyse de régression selon deux modalités un peu particulières. En premier lieu, si la variable dépendante ne change pas, ce sont les notes attribuées dans les différentes disciplines qui vont constituer les variables indépendantes. Sur deux années, celle d'étude plus celle qui précède, le modèle va donc livrer le poids de chaque discipline dans l'accès en seconde de détermination pour un entendement professoral moyen de Melville. Cependant, l'autre différence avec l'utilisation "standard» des modèles logistiques est que nous n'allons pas interpréter directement les coefficients livrés. A la place, nous allons réinjecter le cas de chaque élève de l'année d'étude en cours. Les probabilités livrées sont donc des probabilités théoriques de passage qui, censurées au seuil 0.5 , donnent l'accès théorique ou pas en seconde (sous-entendue: générale et technologique). En les comparant aux passages réels, on va ainsi mettre en évidence des «cas » discordants qu'on pourra analyser à l'aide des matériaux ethnographiques récoltés par ailleurs. Autrement dit, l'analyse de régression logistique fonctionne ici comme un filtre, c'està-dire dans sa dimension pragmatique et non pas analytique.

Dans une logique pragmatique donc, ce sont les fruits et non pas les racines de l'arbre qui nous intéressent ${ }^{6}$. C'est pourquoi nous ne présenterons pas, comme habituellement, le tableau des coefficients de régression, mais seulement les « cas » qu'ils permettent de mettre en évidence. Par exemple, voici les 4 "cas" qui dénotent par rapport à l'évaluation moyenne des résultats par les professeurs de Melville ${ }^{7}$ (tableau 1, page suiv.).

Les deux garçons français n'accèdent pas au lycée général alors que leurs résultats leur permettraient ; les deux filles « étrangères » et «maghrébines » y accèdent alors que leurs résultats scolaires ne leur permettraient pas.

Nous avons donc quatre situations discordantes sur les soixante-seize élèves qui composent ce cycle d'orientation. Mais quel est le poids de chaque discipline dans l'accès dans l'étape postérieure du cursus? Pour le savoir, il nous suffit de faire « travailler » un modèle sans la discipline en question. Plus le nombre de cas non-prédit est important, plus la discipline compte dans le processus d'orientation. Les résultats sont consignés dans le tableau 2 (page suiv.).

Sans surprise, ce sont bien les mathématiques qui restent prépondérantes pour prédire le passage en seconde. Sans elles, nous aurions 7 cas discordants au lieu de 4 .

Par contre, et c'est sur cela que nous allons nous centrer à présent, le poids de l'éducation civique est tout à fait surprenant. Cette discipline pèse autant que la précédente dans le passage en seconde, alors même qu'on peut la considérer comme objectivement anecdotique, comme en témoigne son statut parfaitement flou vis-à-vis de l'histoire-géographie. Comment cela se fait-il ? Pour répondre à cette question, 
regardons plus précisément les élèves qui s'ajoutent aux précédents (tableau 3, page suiv.).

Tableau 1 :

\begin{tabular}{|c|c|c|c|c|c|c|c|c|c|c|c|c|c|c|}
\hline & \begin{tabular}{|l} 
Accès \\
seconde?
\end{tabular} & en & & & & & & 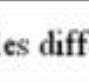 & & & & & & \\
\hline & Théorique & Réel & Mat & $\mathrm{Hg}$ & & Lv1 & Lw2 & Sut & Phe & Eps & $E_{c}$ & $\mathrm{Tec}$ & Ap & $\mathrm{Em}$ \\
\hline $87 \mathrm{Gf}$ & ui & Non & 15,2 & 109 & 11,1 & 12,2 & 15,1 & 15 & 12,7 & 16 & 7,6 & 14,8 & 14,6 & 14,8 \\
\hline $131 G f$ & ii & Non & 13,3 & 16,1 & 16,0 & 15,1 & 159 & 14,6 & 10,6 & 129 & 149 & 14,4 & 12,8 & 139 \\
\hline $110 \mathrm{Fe}$ & Non & ii & 8,3 & 9,6 & 7,7 & 8 & $\mid 11.6$ & 12,4 & 7,8 & 12.3 & 8.6 & 12,7 & 13,8 & 17 \\
\hline $33 \mathrm{Fm}$ & on & Oui & 9.6 & 10,2 & 10,3 & 4,6 & 7,7 & 10.2 & 9.2 & 12.5 & 13,6 & 13,1 & 14,6 & 14 \\
\hline
\end{tabular}

Les élèves sont identifiés, outre le numéro d'index, par un « $G$ » ou un « $F$ » si ils sont « garçon » ou « fille », et par un « $f$ », « $m$ » ou « e » si ils sont classés comme « français », « maghrébin » ou " étranger ». 87Gf est un garçon « français ». Les disciplines sont, dans l'ordre : mathématiques, histoire-géographie, français, langue-vivante 1, langue-vivante 2, science et vie de la terre, physiquechimie, éducation physique et sportive, éducation civique, technologie, arts-plastiques et éducation musicale.

Tableau 2 :

\begin{tabular}{|l|l|}
\hline & Cas discordants \\
\hline \hline Mathématiques & 7 \\
\hline \hline Éducation civique & 7 \\
\hline \hline Français & 5 \\
\hline \hline Langue vivante 1 & 5 \\
\hline \hline E.P.S. & 4 \\
\hline \hline Éducation musicale & 4 \\
\hline \hline Technologie & 4 \\
\hline \hline Langue vivante 2 & 4 \\
\hline \hline Histoire-géographie & 4 \\
\hline \hline SVT & 4 \\
\hline Arts plastiques & 4 \\
\hline \hline Sciences physiques & 4 \\
\hline
\end{tabular}


Tableau 3 :

\begin{tabular}{|c|c|c|c|c|c|c|c|c|c|c|c|c|c|c|}
\hline & $\begin{array}{l}\text { Accès } \\
\text { seconde }\end{array}$ & & & & & & & & & & & & & \\
\hline & Théorique & Réel & Mat & $\mathrm{Hg}$ & Fra & Lv1 & Lv2 & Svt & Phe & Eps & $E_{c}$ & $\mathrm{Tec}$ & Ap & $\mathrm{Em}$ \\
\hline 11 & 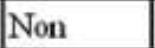 & Oui & 8,0 & 8,4 & 10,4 & 11,0 & 129 & 15,3 & 11,0 & 133 & 12,7 & 13,4 & 11,7 & 15,5 \\
\hline FTe & $I^{*}$ & Oui & 11,0 & 89 & 11,6 & 7,4 & 143 & 12,0 & 10,6 & 11,1 & 139 & 12,6 & 14,5 & 12,3 \\
\hline $6 \mathrm{GI}$ & i & Non & 8,6 & 13,4 & 10,8 & 12,5 & 13,0 & 8,6 & 7,0 & 11,0 & 3,1 & 11,1 & 7,8 & 13. \\
\hline
\end{tabular}

Le garçon «maghrébin » aurait dû accéder en seconde alors que ça n'a pas été le cas ; les deux filles «maghrébine » et « française » y ont accédé alors que ce n'aurait pas dû être le cas. Rapporté au précédent tableau, on a donc trois filles plutôt portées vers la seconde tandis que trois garçons, en vertu de « choix » personnels ou de décisions de l'institution, s'en écartent.

Cela nous fait dire que les filles ont sensiblement été avantagées dans ce cycle d'orientation. Sans pouvoir réellement développer ce résultat (ce n'est pas l'objet de ce texte), il est clair que l'enquête ethnographique abonde en ce sens.

Nous avons assisté aux huit conseils de classes des deux premiers trimestres des quatre classes de troisième du collège Herman Melville. Quel que soit le mode de saisie de ces assemblées, enregistrements ( 7 cas sur 8 ) ou notes ( 1 cas), nous avons toujours été très attentifs aux arguments relatifs au "livret" (les notes proprement dites) et au « dossier » scolaire (les informations extrascolaires considérées comme nécessaires au fonctionnement de l'institution ${ }^{8}$ ). Mais, par dessus tout, nous nous sommes intéressés aux arguments qui ne pouvaient être tirés de ces deux sources. Au-delà donc, ce sont les discussions sur le caractère adéquat ou pas de la cellule familiale qui sont prépondérantes. Or, dans les presque 150 « cas » passés au crible, nous n'avons entendu qu'une seule fois l'idée selon laquelle la situation scolaire d'un jeune garçon pourrait être influencée défavorablement par sa cellule familiale, alors même que ce type d'argument revient plus d'une trentaine de fois pour les jeunes filles. Dans les autres cas masculins (une petite dizaine), l'argumentaire est inverse, à savoir que les familles sont considérées comme "bien méritantes " d'avoir à supporter des jeunes garçons « ingérables ».

En fait, et c'est tout à fait frappant quand on prend un peu de recul, tout se passe comme si la partie la plus sombre du handicap socioculturel, disons la capacité active de la famille à désorganiser l'enfant, ne s'appliquait pas aux garçons. C'est la famille qui pèse sur les jeunes filles tandis que ce sont les jeunes garçons qui pèsent sur leur famille. Cette posture paraît d'autant plus essentialiste pour l'observateur étranger que ce sont en fait certains garçons qui offrent les critères les plus objectifs d'une situation familiale difficile. Ainsi, 103Gf est placé dans un foyer d'accueil pour adolescents. Si son professeur principal voit régulièrement son tuteur, cet état de fait n'a jamais été abordé de quelque manière que ce soit en conseil de classe.

\subsection{De l'orientation à la notation}

Premier constat donc: il existe une discrimination selon le genre au collège Melville. Les jeunes filles semblent ainsi nimbées d'un " être moral » ${ }^{9}$ positif. Quelles que soient leurs conditions objectives d'existence, il semble évident qu'elles mènent une lutte pour s'en affranchir et que l'école reste le moyen privilégié pour le faire. C'est cette bonne volonté scolaire qui est attestée par leurs notes en éducation civique. 
41 Ce type de discrimination, pourtant apparemment hors du cadre que nous nous sommes fixé, reste néanmoins très proche de notre objectif. D'une part car il est une dimension extrêmement importante de la catégorisation opérée par les personnels sur les jeunes issus de l'immigration. Les habitudes discursives ${ }^{10}$ prises pour différencier filles et garçons sont dans un rapport dialectique avec celles liées à l'ethnicité, notamment maghrébine.

[Après un conseil de classe, nous sommes seul avec le principal adjoint dans la salle. Nous faisions remarquer que ce conseil de classe avait été très rapide]

PA: Oui... très très vite parce que heu... c'est une classe homogène, parce que dedans ya des gamins qui bossent... qui ont pas les résultats escomptés mais qui sont dans une perspective heu... correcte... qui sont heu... motivés... et ya... ya des mécanismes à l'intérieur de la classe qui font que même celui qui... ben il est entrâné quand même hein... il est minoritaire et la majorité prend le pouvoir et ya... beaucoup de filles... heu... qui bossent et qui ont des résultats... qui veulent s'en sortir hein... à la maison je bosse hein... donc heu... ma liberté et ben elle passe par là...

Avec « les filles.. heu... qui bossent ", est fait référence à un échange précédant immédiatement la fin du conseil de classe. Sur une demande assez banale de la déléguée de classe d'origine maghrébine (une diminution de la charge de travail à la maison), la fin de non recevoir affichée par le corps professoral s'est subtilement transformée en une investigation à caractère ethnico-religieux. Encouragés par les acquiescements de l'intéressée (sans doute toute heureuse de trouver là un rebond possible d'une affaire qui semblait bien mal engagée), il en a été conclu que la demande, censée pourtant émaner de toute la classe, n'était liée qu'à des difficultés passagères dues à l'augmentation de la charge de travail domestique demandée aux jeunes filles en période de ramadan.

En fait, la figure traditionnelle du boursier trouve ici à s'exprimer par delà le sexe et l'imputation ethnique tant il est vrai que les habitudes discursives contractées à propos des jeunes filles maghrébines posent d'emblée une cellule familiale extrêmement difficile (autoritaire / patriarcale / cloisonnée ; bref : archaïque) dont l'émancipation forcément voulue passe par le rôle libérateur de l'école. En ce sens, la distinction garçon / fille est partie entièrement prenante du procès d'ethnicisation des rapports sociaux dans l'établissement.

D'autre part, car ces orientations discordantes objectivement mal motivées doivent recevoir une justification en contexte. Le cas 121Ff, une des jeunes filles portées vers la seconde, est ici éclairant :

PA : Bien... aller... on passe à... $121 \mathrm{Ff} . .$.

FRA : 121Ff... alors heu... très sérieuse... donc elle, elle demande une seconde générale... mais les résultats sont très moyens... elle va... enfin, elle a des difficultés à comprendre même si ya pas mal de travail...

$[\ldots]$

MATH : Bon, oui : elle fait son travail... comme elle est proche de la moyenne, elle passera... c'est pas le même cas que $118 \mathrm{Fm}$... parce que $118 \mathrm{Fm}$ avait quand même... quand même dans son tableau elle avait des notes mauvaises... là on est tous à peu près... bon, c'est sûr qu'elle va avoir beaucoup de difficultés dans une seconde générale... elle veut pas non plus faire un BEP ?...

Les arguments portés sur le passage ou pas de 121Ff ne prennent tous leur sens qu'à partir du moment où ils sont rapportés au cas contrastif de $118 \mathrm{Fm}$.

[C'est le même conseil de classe que précédemment. Les professeurs et l'encadrement (notamment le professeur de mathématiques et le principal adjoint 
qui dirige la séance) sont les mêmes. Le « cas » 118Fm est analysé avant 121Ff]

PHC : Non mais j'pense qu'y faudrait que... elle envisage... autre chose [qu'une seconde générale ou technologique $118 \mathrm{Fm}]$... enfin... à mon avis hein...

PA : M. MATH, avec 16.38 ?

MATH : Non mais c'est cela... mais seulement quand on dit: on la voit pas en seconde générale avec de telles matières... enfin, de telles notes au premier trimestre et au deuxième trimestre... elle fait appel, elle passe sans problèmes...

LV2 : Oui, mais elle tiendra pas l'coup hein...

MATH : Non mais... attendez... vous ne pouvez pas... ou faut être cohérent un jour ou l'autre... et que les gens disent: bon, moi je vais mettre des notes qui correspondent... qui vont montrer que cette élève n'a pas les moyens... quand je vois les notes qu'elle obtient... 16 heu... 12, 14, 15, 13, 14, 11.8 en français...

PA : À la commission d'appel, ça passe hein... [MATH : C'est même pas la peine de discuter...] ça passe à tous les coups...

Nous ne pouvons pas ici trop nous étendre sur l'ensemble des facteurs congruents qui rendent possible la tournure que prend cet échange. Constatons seulement que, à part ce cas-ci, la validité de la notation est toujours présupposée, toujours évidente, et ce malgré certaines aberrations semblant manifestes. Le fait, par exemple, qu'aucune des notes de français de la $3^{\mathrm{e}} 2$ ne dépasse $7 / 20$ alors qu'elles tournent plus ou moins autour du 10/20 dans les autres classes est immédiatement attribué au « niveau » des élèves, et pas du tout au fait qu'il pourrait s'agir, selon toute vraisemblance, d'une notation particulièrement sévère par rapport à la norme du collège.

C'est dire si le déchirement du voile rhétorique recouvrant la notation auquel nous assistons est tout à fait exceptionnel. Car c'est bien de cela dont il est question ici. De la bouche même d'un des agents, il existe bien à Melville un jeu de la notation. Celle-ci n'est pas tout simplement posée sur les productions de l'élève. Elle «dit " quelque chose et tout l'argumentaire de M. MATH est qu'elle dit bien mal dans le cas présent ce qu'elle est réellement censée dire. Contrairement à sa valeur faciale qui la rend quasiinvulnérable au redoublement et à laquelle le principal adjoint se laisse prendre ( $\mathrm{M}$. MATH, avec 16.38 ?»), l'«essence» de $118 \mathrm{Fm}$ est estimée intuitivement comme inadéquate à l'avenir probable auquel le paradigme évaluatif la destine pourtant.

Si cet «aveu » nous paraît très significatif, il n'est qu'un élément parmi d'autres. L'analyse fine des postures professorales associée au caractère transférable de classe en classe de certaines particularités de notation, les variations inexplicables de performances scolaires des élèves d'une année sur l'autre (tantôt cancres, tantôt doués dans les cas extrêmes), certaines comparaisons avec des notes semblant moins indexées au contexte (notations croisées) nous amènent à conclure qu'il existe effectivement dans le collège Herman Melville d'importants biais sociaux de notation.

Quel est leur impact? En fait, l'ensemble des éléments accumulés à l'intérieur du collège n'est pas l'ensemble des matériaux dont nous disposions. En effet, nous avons avancé un peu plus haut que les biais de notation étaient particulièrement difficiles à mettre en évidence au niveau de l'établissement. Lorsqu'on rapporte les résultats obtenus au collège à un autre établissement moins sensible aux effets de contexte, les lycées par exemple $^{11}$, on peut émettre l'hypothèse qu'une part des particularités dans les conditions sociales de certification apparaissent assez clairement. C'est l'objet de la prochaine section.

4. Le lycée après le collège

Nous avons suivi les élèves du collège Melville dans leur orientation dans le lycée polyvalent du secteur. Quelle est la position de ces élèves quand on les compare aux 
élèves issus des autres collèges précédant le lycée Charles Mingus ? De quelle manière sont-il «marqués » par leur passage à Melville ? Pour les classes de seconde, donc, nous avons collecté les résultats scolaires de l'année précédente, de l'année courante et divisé les lycéens selon leur collège d'origine et leur classe ethnique. En outre, dans la mesure ou nous allions travailler sur les écarts de notation entre collèges et lycée, nous ne nous sommes pas préoccupé plus avant de la classe sociale. Les compétences scripturales-scolaires de chaque élève étant supposées constantes d'une année sur l'autre.

51 Le graphique 1 met en confrontation la valeur médiane ${ }^{12}$ de la moyenne générale des élèves des différents établissements alimentant le lycée. Cette valeur traduit la performance typique des collèges pour l'année en cours (verticalement) et l'année qui précède (horizontalement).

Comme on pouvait s'y attendre et comme on peut le constater en voyant l'éparpillement des points, le paradigme évaluatif reste une norme dont la distance effective à la réalité est importante. Il semble cependant beaucoup mieux respecté dans l'orientation dans la mesure où le seuil médian d'accès en seconde ne varie que sur une plage de 0,7 points. L'entendement professoral moyen de tous les collèges est donc accordé dans un espace inférieur à un point. A gauche, pour les collèges les moins sélectifs donc, nous retrouvons Melville accompagné de Marcel Proust; à droite, le collège Henry Miller est le collège qui demande à ses élèves les performances d'accès en seconde les plus élevées.

53 Cette variation qui peut apparaitre comme relativement minime traduit extrêmement mal les écarts importants quand on s'attache à l'axe des ordonnées. L'écart par rapport aux performances logiquement déductibles des critères d'orientation qu'il matérialise se voit entièrement bouleversé. Ainsi, deux établissements qui étaient très proches du point de vue de leurs sélectivités se retrouvent parfaitement contrastés du point de vue de la situation de leurs élèves en seconde : si les élèves de Marcel Proust semblent ne perdre qu'environ 0,7 point dans la transition vers le deuxième cycle du secondaire, les élèves de Melville perdent quant à eux presque 3,5 points en passant d'un peu plus de 12,5 à un peu plus de 9. L'écart nul entre les deux établissements est ainsi porté à presque trois points. Au-delà de ce cas extrême, le différentiel dans la chute de performance des élèves de tous les collèges à leur arrivée à Charles Mingus nous amène à conclure qu'il existe pour chacun des établissements alimentant le lycée une variable interne qui se manifeste subitement après l'accès en seconde. Apparaissent ainsi des différences importantes qui passeraient parfaitement inaperçues pour une enquête qui s'attacherait à la seule dimension de l'orientation (comme l'enquête de Louis André Vallet et Jean-Paul Caille par exemple). Relativement à notre étude menée à l'intérieur du collège Melville, nous avançons qu'il s'agit de biais sociaux de notation. Quelles en sont leurs conséquences?

Le graphique 2 (page suiv.) montre de manière plus systématique notre constatation faite à Melville, à savoir que la répartition des jeunes issus de l'immigration dans les différents collèges n'est pas équilibrée. 
Graphique 1 :

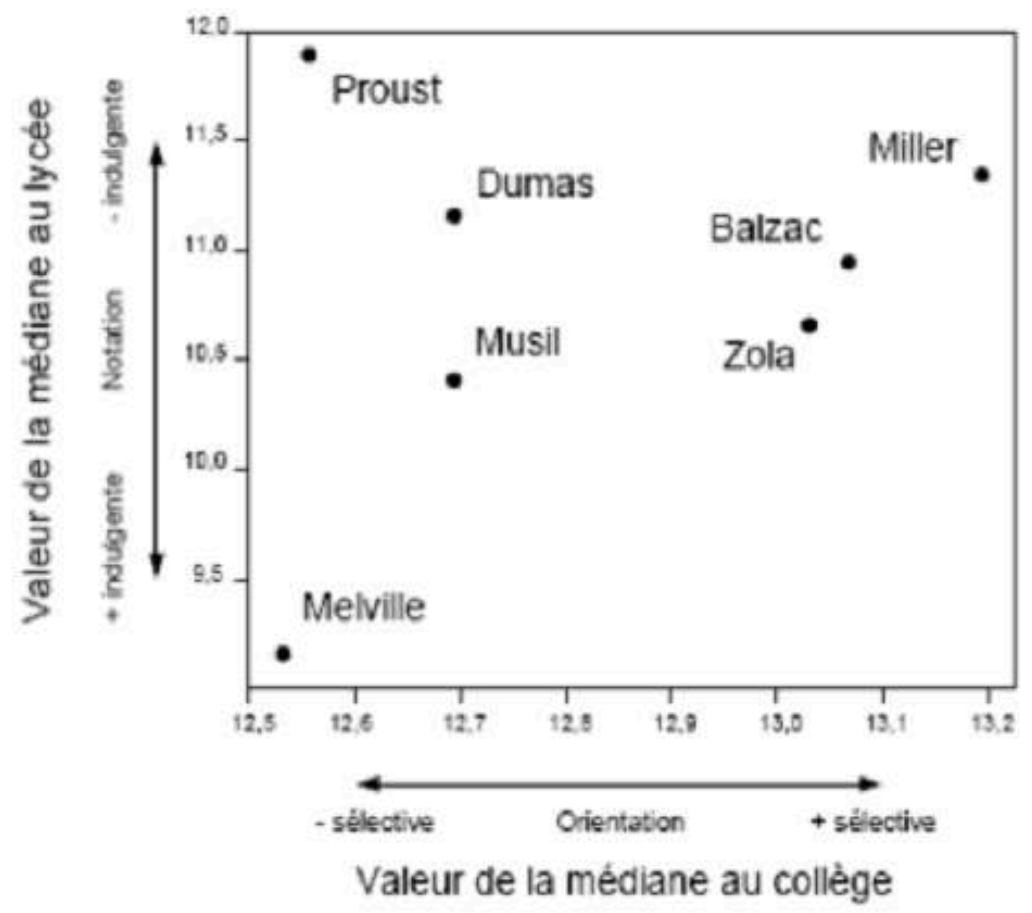

Graphique 2 :

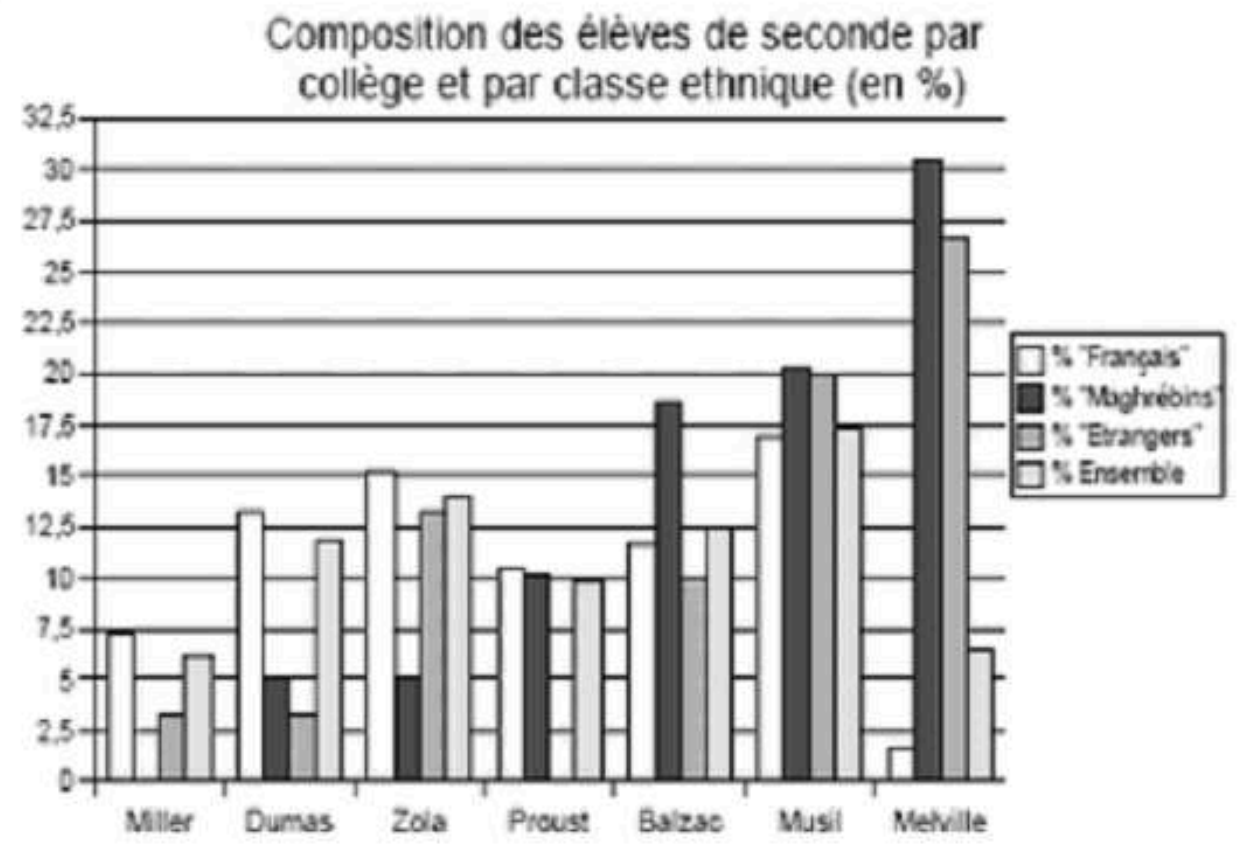

Tableau 4 :

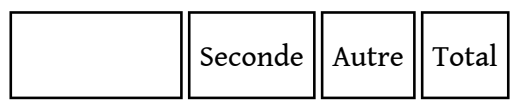




\begin{tabular}{|l|l|l|l|}
\hline Français & 72,2 & 27,8 & 100,0 \\
\hline \hline Maghrébins & 73,1 & 26,9 & 100,0 \\
\hline \hline Étrangers & 75,4 & 24,6 & 100,0 \\
\hline \hline Ensemble & 72,8 & 27,2 & 100,0 \\
\hline
\end{tabular}

Tableau 5 :

\begin{tabular}{|l|l|l|l|}
\hline & Première & Autre & Total \\
\hline \hline Français & 31,8 & 68,2 & 100,0 \\
\hline \hline Maghrébins & 15,3 & 84,7 & 100,0 \\
\hline \hline Étrangers & 20,0 & 80,0 & 100,0 \\
\hline \hline Ensemble & 29,2 & 70,8 & 100,0 \\
\hline
\end{tabular}

Si le collège Melville ne participe que pour environ $7 \%$ des élèves de seconde du lycée Charles Mingus, il participe en revanche pour plus de $30 \%$ des élèves « maghrébins » et pour plus de $25 \%$ des élèves « étrangers ». A eux deux, Melville et Musil, qui n'ont sans doute pas exactement le même profil mais qui restent néanmoins les deux établissements qui ont le moins bien préparé leurs élèves à la seconde, scolarisent plus de la moitié des élèves maghrébins et presque $50 \%$ des élèves étrangers. De fait, toutes les déviations du paradigme évaluatif les concernant, et on sait que l'écart à la norme de Melville est considérable, touche en priorité les jeunes issus de l'immigration.

Pour visualiser très imparfaitement les conséquences de cette ségrégation scolaire, nous avons décidé de comparer les taux de passages théoriques dans le cadre d'une "hypothèse méritocratique stricte " $^{13}$, c'est-à-dire en choisissant un seuil à partir duquel l'accès dans la classe supérieure serait automatique. A rebours donc, à 12/20 pour l'accès en seconde, nous obtenons les données présentées dans le tableau 4 (page précédente).

Un peu plus d'un quart des élèves de seconde n'avaient pas au collège une moyenne supérieure à $12 / 20$. Cette proportion varie assez peu selon les classes ethniques, avec un très léger avantage même pour les élèves « étrangers » et " maghrébins ». Comme on l'a dit, le processus d'orientation est plutôt cohérent.

Avec le même seuil au lycée pour le passage de la seconde à la première (tableau 5, page précédente), le taux de passage global chute à un peu moins de $30 \%$, et la différence entre les classes ethniques s'est considérablement creusée. Ainsi, plus de 15 points séparent les élèves "maghrébins" des élèves «français». Même si les élèves " étrangers » semblent mieux lotis, cet écart est encore de plus de 10 points. Dans tous les cas, on rappelle également que toutes les premières ne se valent pas et que la prise en compte des filières accentue encore (et de beaucoup) le phénomène constaté. 


\section{Conclusion}

61 Si la vertu heuristique d'un concept comme la discrimination devait rester liée à sa capacité intrinsèque de désignation directe, la seule "discrimination » que nous aurions pu mettre en évidence à l'intérieur de Melville est celle qui sépare les filles des garçons. C'est la seule en effet qui s'établit à partir d'un critère supportant un mécanisme d'inclusion / exclusion fonctionnant a priori.

Ainsi, sans vraiment l'avoir cherché, nous sommes littéralement «tombé » dessus. Cela se comprend. Cette discrimination offre en effet tous les critères traditionnels, classiques, de lisibilité. Elle prend place au sein de moments de la vie sociale, les conseils de classe, qui sont une activité dont le programme, les manières et le temps de réalisation semblent très formalisés et très rationalisés ${ }^{14}$. Elle repose sur un critère qui semble quasiment univoque (le sexe) et a des conséquences qui sont statistiquement très claires. Enfin, cerise sur le gâteau du point de vue du nouveau paradigme, elle est accompagnée d'une enveloppe discursive dont les ressorts sont familiers.

Par contre, même sans prendre en compte ce dernier critère, chercher à trouver une figure semblable dans le domaine ethnique aurait été parfaitement vain.

La discrimination que nous avons mise en évidence est temporellement distendue. Elle a sans doute quelque chose à voir avec l'idée de moment, mais uniquement dans une acception systémique: aucun de ces moments n'a de sens en lui-même; tous n'acquièrent leur signification que lorsqu'ils sont replacés dans le réseau d'interdépendance qui les lie. Ainsi, on ne «voit " réellement l'orientation en seconde qu'à partir du moment où on "voit " l'orientation en première; la notation au collège qu'à partir du moment où on "voit » celle du lycée ; l'orientation quand on « voit » la notation ; et ainsi de suite...

65 Enfin, on ne peut comprendre la situation des enfants de migrants qu'à partir du moment où, à un critère censé résumer l'alpha et l'oméga de la discrimination, on y substitue une constellation de facteurs. Sans doute, l'intentionnalité raciste, sécularisée ou pas, y a une place. Mais elle ne constitue pas la formule encastrée dans les actes permettant finalement de déduire en ligne directe un différentiel de position. Ainsi, à défaut d'un facteur plus franchement lié à l'orientation ou à la notation, le « refus de cohabiter $»^{15}$ qu'on lit sans peine dans la ségrégation scolaire n'a aucun lien privilégié avec le constat final de la discrimination. Sans sa connexion avec les facteurs précédemment cités son impact est, à vrai dire, quasi-nul.

66 Ceci précisé, la discrimination que nous avons mise en évidence n'en est pas moins une au sens plus ou moins usuel du terme. Elle s'en distingue mais ne s'en sépare pas. De sorte qu'elle ne mérite sans doute pas d'être simplement appelée une " conséquence » de la ségrégation scolaire.

67 En effet, notre travail nous a conduit à mettre en évidence la faible portée des « effets de contexte » sur la valeur faciale d'un établissement telle qu'elle peut se présenter à l'observateur ne prenant en compte que des données institutionnelles. De sorte que, comme monde relativement autonome, Herman Melville peut apparaître comme aussi « normal » que possible. Sans doute, comme aime à le rappeler son encadrement, le "handicap culturel» fait qu'il occupe une place assez basse dans la hiérarchie institutionnelle. Mais celle-ci apparaît néanmoins comme cohérente avec sa population. Les taux de passage en seconde sont ainsi faibles, mais pas ridiculement faibles au point de remettre en cause la logique même du processus d'orientation. 
ce dernier offre déjà son lot de tensions relatives à l'inflexion de certaines trajectoires vers l'enseignement professionnel. Certaines de ces tensions trouvent leur expression chez les élèves au travers l'imputation de motifs liées à des comportements discriminatoires. Ainsi, il n'est pas rare de rencontrer des propos selon lesquels c'est parce que "l'école» / "le collège » / « les Français » n'aiment pas les « Arabes » que ces derniers sont orientés vers le BEP. Mais ce n'est pas parce que nous n'avons pas pu mettre en évidence un tel comportement que l'on peut disposer de tels propos en les opposant aux «faits» et en les traitant comme de «simples» représentations. A condition qu'on leur associe un contexte de description pertinent (c'est le travail du sociologue), on est forcé de les prendre au sérieux. Ainsi la confrontation de l'année de seconde avec celle de troisième montre qu'ils sont objectifs.

Dans le cadre de l'induction normale, c'est parce que le collège est socio-ethniquement homogène que les orientations en lycée professionnel sont aussi nombreuses, aussi visibles, et aussi facilement imputables à des critères ethniques. Or, c'est en effet cette homogénéité qui fait porter en priorité sur les jeunes issus de l'immigration les conséquences de la sédimentation des biais de notation. La discrimination perçue est donc bien réelle, mais elle s'exprime en quelque sorte à contre temps et par anticipation.

Ce caractère hors phase a néanmoins des effets pour l'actualisation des rapports de force. Il permet d'externaliser une part des recoupements catégoriels susceptibles de condenser sur le collège des principes d'actions et de revendications. Les taux de passage sont ainsi beaucoup moins rugueux que s'ils avaient dû correspondre au paradigme évaluatif. Arrivé en seconde générale et obligé tout d'un coup de faire face à son niveau réel, l'élève " imméritant » n'est plus du tout supporté par le même univers de sens. Dilué dans le lycée, disposant d'un environnement bien moins riche en reconnaissances et solidarités immédiates, c'est probablement à lui-seul qu'il devra imputer son relatif échec, celui du redoublement et de l'orientation (ou de la réorientation) vers les filières les moins nobles.

\section{BIBLIOGRAPHIE}

BOUVERESSE Jacques (1995), « Règle, disposition, et habitus ». Critique, n579-580, pp. 573-594.

COUSIN Olivier (1996), « Construction et évaluation de l'effet établissement : le travail des collèges ». Revue française de pédagogie, $\mathrm{n}^{\circ} 115$, pp. 59-75.

DE RUDDER Véronique, POIRET Christian et VOURC'H François (2000), L'inégalité raciste. L'universalité républicaine à l'épreuve. Puf, Paris.

DURU-BELLAT Marie et MINGAT Alain (1993), Pour une approche analytique du système éducatif. Puf, Paris.

FELOUZIS Georges (2003), « La ségrégation ethnique au collège et ses conséquences ». Revue française de sociologie, $n^{\circ} 3$, pp. 413-447.

Cahiers de I'Urmis, 10-11 | 2006 
GARCIA Sandrine et POUPEAU Franck (2003), « La mesure de la "démocratisation scolaire". Notes sur les usages sociologiques des indicateurs statistiques ». Actes de la recherche en science sociale, $n^{\circ} 149$, pp. $74-87$.

LAHIRE Bernard (2005), L'invention de l'« illettrisme » : rhétorique publique, éthique et stigmates. La Découverte, Paris.

LAHIRE Bernard (2000), Culture écrite et inégalités scolaires. Pul, Lyon.

MAETZ Isabelle (2005), « Ségrégation ou mixité : la répartition des élèves dans les collèges et les lycées ». Note d'information, 05.37, Paris, MENSR.

MERLE Pierre (1996), L'évaluation des élèves. Enquête sur le jugement professoral. Puf, Paris.

NOVI Michel et ZIROTTI Jean-Pierre (1979), La scolarisation des enfants de travailleurs immigrés. Vol. 1 : Evaluation, sélection et orientation scolaire. Ideric, Nice.

PASSERON Jean-Claude (1982), « L'inflation des diplômes. Remarques sur l'usage de quelques concepts analogiques en sociologie ». Revue française de sociologie, $n^{\circ} 4$, pp. 551-584.

R Development Core Team (2005), R: A Language and Environment for Statistical Computing. $\mathrm{R}$ Fondation for Statistical Computing, Vienne, http://www.R-project.org.

SIMON Patrick (2005), Le rôle des statistiques dans la transformation du système de discrimination, http://seminaire.samizdat.net/IMG/pdf/Patrick_Simon_2.pdf.

VALLET Louis-André et CAILLE Jean-Paul (1996), « Les élèves étrangers ou issus de l'immigration dans l'école et le collège français. Une étude d'ensemble », Les dossiers d'éducation et formation, $n^{\circ} 67$.

ZIROTTI Jean-Pierre (1984), La scolarisation des enfants de travailleurs immigrés. Les mécanismes institutionnalisés de la domination : processus objectifs et effets subjectifs. Ideric, Nice.

\section{NOTES}

1. Lahire (2000)

2. Maetz (2005)

3. Felouzis (2003)

4. Cousin (1996)

5. Comme toutes les catégories employées en cours de recherche, celles-ci ont comme caractéristique essentielle d'être commodes, c'est-à-dire d'être un compromis opératoire entre tout un ensemble de contraintes tant théoriques que pratiques (méthodologiques) qu'il serait trop long d'exposer ici. Inutile donc de dire qu'elles ne présument pas d'une éventuelle substance racio-ethno-culturelle.

6. Nous nous méfions terriblement de l'idée selon laquelle la mécanique du modèle, la somme de ses moments, serait en charge de nous révéler « la mécanique sociale en fonctionnement » comme le dit Jacques Bouveresse (1995).

7. L'ensemble des traitements statistiques ont été réalisés avec GNU R. GNU R est une version logiciel libre (librement diffusable et librement modifiable) du dialecte " S Plus ", à la base de certains logiciels propriétaires comme SAS. Cf. R Core Team (2005).

8. Lieu de résidence, profession des parents, inscription à la demi-pension, etc.

9. Novi et Zirotti (1979)

10. Lahire (2005) 
11. «Le fait que le secteur de recrutement d'un lycée soit plus grand que celui d'un collège réduit les différences entre lycées par rapport à celles des collèges. », Maetz (2005), p. 3.

12. Pour rappel, la valeur médiane est la valeur qui coupe un échantillon en deux parts égales. Une médiane de 12,5 signifie que $50 \%$ des élèves on une moyenne générale supérieure et $50 \%$ inférieure. Cet indicateur a été choisi dans la mesure où il se rapproche plus de l'idée de seuil que, par exemple, la moyenne (la moyenne des moyennes dans le cas présent).

13. Novi et Zirotti (1979)

14. Le testing à l'embauche construit ce type de moment. L'utilisation présupposée universelle de la méthode expérimentale et du raisonnement « toutes choses égales par ailleurs » qu'elle implique fabrique une procédure d'embauche dont la lisibilité procédurale tend à être considérée comme acquise sur une large échelle. Le testing fait ainsi l'impasse sur les embauches qui n'ont pas fait l'objet d'une offre publique et celles qui, malgré cette offre, se basent sur des critères de confiance plutôt que de compétence (capital social). C'est ainsi que les fréquences de rejet des deux CV présentés, ainsi qu'une estimation des cas où ils n'ont pas pu être présentés, sont rarement l'objet d'une attention particulière.

15. De Rudder et al (2000)

\section{AUTEUR}

\section{PHILIPPE PERROT}

Doctorant Urmis-Soliis, université de Nice Sophia-Antipolis 